\title{
Collapse transitions of a periodic hydrophilic hydrophobic chain
}

\author{
E. Orlandini and T. Garel \\ Service de Physique Théorique \\ CE-Saclay, 91191 Gif-sur-Yvette Cedex, France
}

(October 27, 2018)

Accepted for publication in:

Saclay, SPhT/98-022

"Eur. Phys. J. B"

PACS: 61.41.+e; 87.15.-v; 64.70.-p

Short title: Transitions of a periodic hydrophilic hydrophobic chain 
We study a single self avoiding hydrophilic hydrophobic polymer chain, through Monte Carlo lattice simulations. The affinity of monomer $i$ for water is characterized by a (scalar) charge $\lambda_{i}$, and the monomer-water interaction is short-ranged. Assuming incompressibility yields an effective short ranged interaction between monomer pairs $(i, j)$, proportional to $\left(\lambda_{i}+\lambda_{j}\right)$. In this article, we take $\lambda_{i}=+1$ (resp. $\left(\lambda_{i}=-1\right)$ ) for hydrophilic (resp. hydrophobic) monomers and consider a chain with (i) an equal number of hydro-philic and -phobic monomers (ii) a periodic distribution of the $\lambda_{i}$ along the chain, with periodicity $2 p$. The simulations are done for various chain lengths $N$, in $d=2$ (square lattice) and $d=3$ (cubic lattice). There is a critical value $p_{c}(d, N)$ of the periodicity, which distinguishes between different low temperature structures. For $p>p_{c}$, the ground state corresponds to a macroscopic phase separation between a dense hydrophobic core and hydrophilic loops. For $p<p_{c}$ (but not too small), one gets a microscopic (finite scale) phase separation, and the ground state corresponds to a chain or network of hydrophobic droplets, coated by hydrophilic monomers. We restrict our study to two extreme cases, $p \sim O(N)$ and $p \sim O(1)$ to illustrate the physics of the various phase transitions. A tentative variational approach is also presented. 


\section{INTRODUCTION}

A very popular approach to the protein folding problem, is to emphasize the heterogeneity of a protein due to the different side chains [1]. Of special importance in this context is the model of a (quenched) random hydrophilic hydrophobic chain, since (i) proteins are usually designed to work in water (ii) the first step of the folding transition may correspond to the formation of a hydrophobic core [2]. In this model, each monomer is described by a single "charge" $\lambda_{i}$, and the polymer-water interactions are modelled through the Hamiltonian:

$$
\mathcal{H}_{p w}=-\sum_{i=1}^{N} \sum_{\alpha=1}^{\mathcal{N}} \lambda_{i} a\left(\vec{r}_{i}-\vec{R}_{\alpha}\right)
$$

where $a(\vec{r})$ denotes a short-ranged (Van der Waals-like) monomer-water molecule interaction, $N$ and $\mathcal{N}$ denote respectively the number of monomers and of water molecules, and $\vec{r}_{i}$ and $\vec{R}_{\alpha}$, their respective positions. Assuming that the system is incompressible, one gets [1, 3]

$$
\mathcal{H}_{p w}=+\frac{1}{2} \sum_{i=1}^{N} \sum_{j=1}^{N}\left(\lambda_{i}+\lambda_{j}\right) a\left(\vec{r}_{i}-\vec{r}_{j}\right)-A \sum_{i=1}^{N} \lambda_{i}
$$

where $A=\sum_{\vec{r}} a(\vec{r})$. The second term in (2) is a constant for the quenched disordered chain (as well as for the periodic chain studied in this article). It will therefore be omitted henceforth. The phase diagram of this random hydrophilic hydrophobic polymer has been studied by various methods (mean-field, dynamics, replica variational calculations,....) for simple disorder distributions of the $\lambda_{i}$ 's [4, 5, 66,7]. The results can be roughly summarized as follows

(i) for strongly (on average) hydrophobic chains, one expects a $\theta$ collapse transition to occur first, followed at lower temperatures, by a scale dependent freezing transition.

(ii) for chains that are (on average), either weakly hydrophobic or hydrophilic, one expects a freezing transition that is perhaps characterized by a "random first order" transition 8.

An interesting application of this model to proteins has recently been published [9], even though their hydrophobic content appears not to be random [10. 
As a first step towards the protein folding problem, we will study numerically a single self avoiding chain, where the distribution of the $\lambda_{i}$ 's is periodic along the chain. To be more specific, the chain of $N$ monomers is made out of periodically alternating blocks of $p$ hydrophilic monomers $\left(\lambda_{i}=+1\right)$ and $p$ hydrophobic monomers $\left(\lambda_{i}=-1\right)$. Following equation (2), the Hamiltonian of the chain is defined as

$$
\mathcal{H}=+\frac{1}{2} \sum_{i=1}^{N} \sum_{j=1}^{N}\left(\lambda_{i}+\lambda_{j}\right) \Delta\left(\vec{r}_{i}-\vec{r}_{j}\right)
$$

where $\Delta\left(\vec{r}_{i}-\vec{r}_{j}\right)$ denotes a lattice $\Delta$ function: $\Delta(\vec{r})=1$, if $\vec{r}$ connects nearest neighbours, and 0 otherwise. In this paper, we will consider square $(d=2)$ and cubic $(d=3)$ lattices.

The parameter $p$ is an important ingredient of the problem since there is a critical value of $p$, that we note $p_{c}(d, N)$, above which the equilibrium ground state consists of a single hydrophobic core. The argument is as follows; for a spherical core $(d=3)$, the number of points on the surface is of order $N^{\frac{2}{3}}$, whereas the surrounding hydrophilic loops occupy a number of surface points of order $\frac{N}{p}$. Therefore, one expects a single macroscopic hydrophobic core for $p>O\left(N^{\frac{1}{3}}\right)$, and many microscopic hydrophobic droplets for $p<$ $O\left(N^{\frac{1}{3}}\right)$. We therefore get $p_{c}(3, N) \sim O\left(N^{\frac{1}{3}}\right)$. For $d=2$, the steric constraints are much stronger, and one hydrophilic loop anchored on the hydrophobic core screens part of its "surface". Since the distance between the two ends of the hydrophilic loop typically scales with the radius of gyration of the core, that is also with its "surface", this screening effect is very strong. It implies that $p_{c}(2, N) \sim O(N)$. In the case $p<p_{c}(d, N)$, corresponding to many hydrophobic droplets, one should further distinguish between a linear or branched topology of the hydrophobic droplets. For very small $p$, the very formation of these droplets is impeded by the hydrophilic parts of the chain (see below)

The numerical simulations for the periodic chain of equation (3) are done on the square or cubic lattice, using the Multiple Markov chain method [11]. The plan of the paper is

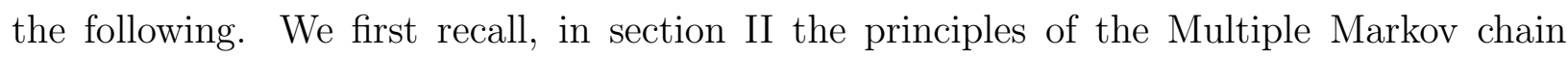
method in Monte Carlo simulations of polymer chains. As a preliminary test, we apply this method to the two dimensional $\theta$ collapse of a purely hydrophobic chain. We then consider 
the periodic hydrophilic hydrophobic chain in two extreme cases, namely $p \sim O(N)$ and $p \sim O(1)$, to capture the physics of the various phase transitions. Putting numbers on the above estimates of $p_{c}$ shows that it will be numerically convenient to study the single core phase in $d=3$ and the multiple cores' phase in $d=2$. In section $[1]$, the case $p \sim O(N)$ is studied in detail for $d=3$; we also briefly consider the corresponding transition in $d=2$. In section IV, we study the case $p \sim O(1)$ which, as stated above, has a multiple droplet low temperature structure: for $d=2$, we present evidence for the existence of an intermediate branched phase, if $p$ is not too small. The $d=3$ case requires very long chains and is only briefly considered. Finally, we also present a tentative variational method for this case.

\section{THE MULTIPLE MARKOV CHAIN METHOD}

\section{A. Summary of the method}

In this section we give a quick description of the numerical techniques which we use to calculate thermodynamic (and/or geometric) properties of the chains as a function of the temperature. These techniques have been discussed in detail in reference [12], and can be summarized as follows.

The implementation of the Metropolis Monte Carlo method relies on the multiple Markov chain sampling. First, one generates with the simple Metropolis heat bath, a Markov chain at temperature $T$ : this procedure makes use of an hybrid algorithm, which has pivot 13 as well as crankshaft moves [14]. Pivot moves are of a global type, and operate well in the swollen phase; crankshaft moves, which are of a local type, are essential in speeding up convergence close to the collapse phase transitions [12]. In these calculations, each Monte Carlo step consists of $O(1)$ pivot move and $O(N)$ crankshaft moves.

One may then run in parallel a number $m$ (typically $15-20$ ) of these Markov chains at different temperatures $T_{1}>T_{2}>\ldots>T_{m}$. We allow the chains to interact (by possibly swapping conformations) as follows. Among the $m$ chains, we select, with uniform proba- 
bility, two chains $(\alpha, \beta)$ at respective temperature $T_{\alpha}$ and $T_{\beta}$. A trial move is an attempt to swap the two current conformations of these chains. If $\pi_{K}(T)$ is the probability of getting state $K$ at temperature $T$ (that is $\pi_{K}(T) \sim e^{-\frac{H(K)}{T}}$, in obvious notations), and $S_{\alpha}$ and $S_{\beta}$ are the current states in the $\alpha$-th and $\beta$-th chain, then we accept the trial move (i.e. we swap $S_{\alpha}$ and $S_{\beta}$ ) with probability

$$
r\left(S_{\alpha}, S_{\beta}\right)=\min \left(1, \frac{\pi_{S_{\beta}}\left(T_{\alpha}\right) \pi_{S_{\alpha}}\left(T_{\beta}\right)}{\pi_{S_{\alpha}}\left(T_{\alpha}\right) \pi_{S_{\beta}}\left(T_{\beta}\right)}\right) .
$$

Note that the whole process is itself a (composite) Markov chain. Since the underlying Markov chains are ergodic, so is the composite Markov chain. Furthermore, the composite chain obeys detailed balance, since the swap moves as well as the moves in the underlying chains obey detailed balance [12]. The swapping procedure dramatically decreases the correlations within each Markov chain, and produces little CPU waste since, in any case, one is interested in obtaining data at many temperatures [11].

\section{B. The two dimensional $\theta$ transition}

We first consider the application of the Multiple Markov chain method to the two dimensional $\theta$ collapse transition of a purely hydrophobic chain (see ref [12] for the case $d=3$ ). We are mostly interested here in locating the thermodynamic $\theta$ transition.

We show in Figure 1 the plot of the average scaled radius of gyration $\left(\frac{<R^{2}>}{N^{2 v_{\theta}}}\right)$ versus temperature, for different values of $N$. Finite size scaling theory [15, 16, 17, 18, 19, 20] predicts that

$$
\frac{<R^{2}>}{N^{2 \nu_{\theta}}}=f\left(t N^{\Phi}\right)
$$

where $t=\left|\frac{T-T_{\theta}}{T_{\theta}}\right|$ is the reduced temperature for $N$ large $\left(T_{\theta}=T_{\theta}(N=\infty)\right), \Phi$ a crossover exponent, and $f(x)$ a scaling function with a finite value for $x \rightarrow 0$. Using the exact result

$2 \nu_{\theta}=\frac{8}{7}$ [21] yields an estimate of the $\theta$ temperature: $T_{\theta} \simeq 1.5$, in agreement with recent estimates [19]. To study the critical behaviour of the specific heat close to the $\theta$ point is a 
notoriously difficult problem in $d=2$. We have also verified this point, and we have only extracted from the $N$ dependence of the peak of the specific heat (Figure 2) a large $N$ critical temperature $T_{\theta}^{\prime} \sim 1.5$ in broad agreement with the value obtained from the behaviour of the radius of gyration.

\section{THE CASE $P=O(N)$}

\section{A. Numerical simulations for $d=3$}

This case corresponds to a single hydrophobic core ground state since $p_{c}(3, N) \sim O\left(N^{\frac{1}{3}}\right)$. In the case of the $\theta$ collapse transition, the $N$ monomers of the chain play an equivalent role (neglecting end effects for a long enough chain). In the present problem, the repulsive interaction between hydrophilic monomers leads us to consider two possibilities (i) the collapse transition first occurs in a single hydrophobic block of length $p$ (ii) the collapse is due to a cooperative effect of the $\frac{N}{2 p}$ hydrophobic blocks, in a way similar to the $\theta$ transition. Scenario (ii) is consistent with a unique phase transition of a discontinuous character occurring at

temperature $T_{\theta}\left(\frac{N}{2}\right)$. Scenario (i) is a priori consistent with a collapse transition at $T_{\theta}(p)$, and raises the question of a (surface induced) sticking transition of the individual hydrophobic blocks.

In either case, the collapse transition is expected to be discontinuous, with a jump of the radius of gyration. We have fixed $p=\frac{N}{8}$ in the simulations, and let $N$ vary from 80 to 640 . A typical low temperature configuration for $N=640$ is shown in Figure 3: as expected, it displays a macroscopic phase separation between the hydrophobic and hydrophilic parts of the chain. To get an estimate of the critical temperature, we have first considered the radius of gyration $<R^{2}>$ of the complete chain. As shown in Figure 4, the exponent of $<R^{2}>$ is, at all temperatures, given by the self avoiding walk (SAW) value $\left(\nu_{S A W} \simeq 0.588\right.$ [22,23]).

Our data for the large values of $N$ are in agreement with scenario (i), that is a single hydrophobic block collapse, since the critical temperature $T_{c}$ is very close to $T_{\theta}(p)$ (see Table 
I). A better estimate of $T_{c}$ comes from specific heat measurements, since the specific heat has a quite spiky character (Figure 5), unlike its $\theta$ point equivalent [12]. We again get an estimate very close to $T_{\theta}(p)$. We have also tried a finite size scaling analysis to find $T_{c}$, for large $N$ (and therefore large $p$ ). Following a well established path (see e.g. [24] and references therein), we have considered the $N$ dependence of the height and position of the peak in the specific heat. At the transition, and in the scaling limit, one expects the height peak $C^{*}$ to scale like

$$
C^{*} \sim N^{2 \Phi-1}
$$

where the crossover exponent $\Phi$ and the specific heat exponent $\alpha$ are related [25] through the relation $\Phi(2-\alpha)=1$. At the three dimensional $\theta$ transition, one has $\Phi=0.5$ and $\alpha=0$. At a (thermal) first order transition, one has $\alpha=1$, yielding $C^{*} \sim N$. Finite size scaling also implies a critical temperature shift $\Delta T_{c}=T_{c}(\infty)-T_{c}(N) \sim \frac{1}{N^{\Phi}}$, yielding $\Delta T_{c} \sim \frac{1}{N}$ for a first order transition. Figures 6(a) and 6(b) show our results, and confirm that our simulations are not done in the scaling regime. It is well known indeed that, in order to get a precise estimate of the thermodynamic $\theta$ temperature, one has to study very long chains (typically $N>1500$ ). For our problem, since $p=\frac{N}{8}$, we should study the case $N=12000$, which is presently out of reach. We have done an independent simulation with $N=800$, and $p=\frac{N}{4}=200$. The peak in the specific heat occurs for $T_{c} \simeq 2.5$, very close with $T_{\theta}(p=200)$ (see Table I).

We therefore believe that, in the thermodynamic limit $(N, p \rightarrow \infty)$, one has a discontinuous single block collapse transition; the critical temperature $T_{c}(\infty)$ is the same as the collapse temperature of the fully hydrophobic chain $\left(T_{\theta}=T_{\theta}(\infty) \simeq 3.7[20]\right)$. The transition is well characterized by the phase separation order parameter [6:7]:

$$
<\delta R^{2}>=<R_{p h i l}^{2}>-<R_{p h o b}^{2}>
$$

where $<R_{\text {phil }}^{2}>$ (resp. $<R_{\text {phob }}^{2}>$ is the squared radius of gyration of the hydrophilic (resp. hydrophobic) monomers. In Figure 7 , we have plotted the order parameter $\left(\frac{<\delta R^{2}>}{N^{2 \nu} S A W}\right)$ 
as a function of temperature, for various values of $N$. Its behaviour is consistent with the previous results.

An intriguing feature of the single block collapse mechanism is that it seems to imply the existence of an intermediate phase, between the low temperature phase depicted in Figure 3 , and the swollen coil phase. This intermediate phase is a necklace (or network) of single hydrophobic blocks, and its free energy differs from the low temperature free energy by a surface free energy. We have not found this intermediate phase in our multiple chain Monte Carlo Method, and this may have several causes. It is for instance possible that the temperatures of the various chains $T_{1}>T_{2}>\ldots>T_{m}$ of our simulations had too large a spacing to find this phase. Further work, in particular a precise determination of surface properties, is needed on this point.

\section{B. Numerical simulations for $\mathrm{d}=\mathbf{2}$}

In this case, one expects $p_{c}(2, N) \sim O(N)$, yielding two different situations. If $p>p_{c}$, one will get a single hydrophobic core below the collapse transition (Figure 8). One may then study the (full) radius of gyration at high and low temperatures (Figure 9), obtaining

in both cases self avoiding behaviour (i.e. $\nu=\frac{3}{4}$ [26]). If $p<p_{c}$, several hydrophobic cores appear (Figure 10). On the square lattice, we have found numerically that $0.06<$ $p_{c}(2, N) / N<0.08$. One may get a feeling for this result by considering a square shaped hydrophobic single core that is fully surrounded by hydrophilic loops: the above estimates corresponds to a total number of hydrophilic loops approximately equal to $6-8$. This argument is only suggestive since the single core becomes elongated as $p \rightarrow p_{c}^{+}$. This is clearly due to the screening effect of the hydrophilic loops: the hydrophobic core tries to maximize its perimeter at fixed surface (Figure 11). As for the phase transition for $p>p_{c}$, one may say that specific heat data display a rather smooth behaviour; the transition is not markedly discontinuous. As already mentionned, extracting a more detailed information from these data is rather tricky in two dimensions. 


\section{THE MANY HYDROPHOBIC DROPLET CHAIN $\mathrm{P}=\mathrm{O}(1)$}

\section{A. Numerical simulations for $\mathrm{d}=\mathbf{2}$}

The large value of $p_{c}(2, N)$ shows that the multiple droplets' phase should be a priori easier to study in two dimensions. We show in Figure 12 a typical low temperature configuration, which displays branched polymer features [27]. From section IIIB, we know that the maximum number of hydrophobic monomers in a droplet is $n_{M A X} \sim 12-16 p$. To further investigate the branched character of the phase, we have studied the case $p=8$, with $N$ ranging from 80 to 1200 (other values of $p$ are briefly considered below). For $p=8$, the high and low temperature exponents $\nu$ of the radius of gyration are shown on Figure 13. Above the transition, we get SAW behaviour; below the transition, we get $\nu \simeq 0.64$, which is indeed close to the branched polymer (BP) value [28].

It turns out that low temperatures are difficult to study because of non-equilibrium effects, so that we are not able to follow in detail the thermal evolution of the branched phase. This is partially due to the fact that the Monte Carlo method of section \ has not been optimized to deal with branched phases. Another reason may be the possible existence of a dynamical phase transition towards some kind of glassy branched state (see section IVQ). We have therefore restricted our study to the phase transition between the high temperature (SAW) and low temperature (BP) phases. Using the same finite size argument

as in section [1B, we plot in Figure 14 the scaled radius of gyration $\left(\frac{<R^{2}>}{N^{2 \nu^{*}}}\right)$ vs temperature. Various values of the unknown exponent $\nu^{*}$ have been considered (Figure 15). Our results show the existence of a phase transition at $T_{c} \simeq 0.8 \pm 0.1$, and evidence for a new critical behaviour $\left(\nu^{*} \simeq 0.70 \pm 0.03\right)$ at $T_{c}$. The phase transition seems also to be quite smooth, if one considers specific heat data. Another "experimental" observation concerns the size distribution of the hydrophobic droplets: below the transition, we find that most of the droplets do not reach the maximum size allowed $n_{M A X}$. This can be interpreted as an entropic effect, very much along the lines of reference $[$ ]. 
We conclude this section by a few remarks on the role of $p$. We have also considered the case $p=4$, and $p=10,12$, with the same range of values of $N$. We do not find a clear evidence for a phase transition for $p=4$, whereas we find evidence for two transitions for $p=10,12$. In the latter case, the branched phase gives way at low temperature to a reentrant self avoiding chain of finite hydrophobic droplets. This shows that the balance between linear and branched topologies is very dependent on the value of $p$. If $p$ is too small, the formation of the droplets is impeded by the repulsive hydrophilic monomers. For large $p$, a local collapse is possible, favoring the linear topology at low temperature. For intermediate values of $p$, a non local hydrophobic collapse is apparently favored, yielding a branched topology. These issues will be further tackled in section IVQ.

\section{B. Numerical simulations for $\mathrm{d}=3$}

A typical low temperature configuration is shown in Figure 16 , for $p=4, N=720$. The properties of the multiple hydrophobic cores phase are difficult to study, since one needs very large values of $N$. Furthermore, for $d=3$, a SAW at the $\theta$ point and a branched polymer (BP) have the same exponent $\nu_{\theta}=\nu_{B P}=0.5$ [29], which makes a detailed scaling analysis difficult.

\section{Variational method for the many droplet phase}

Following traditional polymer physics methods [1], we will study the low temperature branched phase in a variational way. The basic steps can be summarized as follows:

(i) one derives an effective quantum Hamiltonian.

(ii) ones uses a ground state approximation, together with a saddle point approximation.

(iii) finally, one performs a variational calculation, and minimizes the free energy with respect to the relevant parameters.

Steps (i) and (ii) are familiar in the context of the usual $\theta$ collapse transition. Since one is then interested in a macroscopic collapse, a continuous description of the chain is valid; 
furthermore, the ground state approximation holds for long enough chains, since there is a bound state representative of the collapsed globule. Finally, step (iii) is usually implemented with a constant or Gaussian density around the center of mass of the collapsed globule, which can be taken as fixed in all calculations.

On the contrary, what we have in the low temperature branched phase is a inhomogeneous liquid of microscopic hydrophobic droplets. If one follows the above procedure, one must take the extensive entropy of these droplets (i.e. the degeneracy of the saddle point) into account. Since we believe that our approach may be of interest in other contexts [8], we will assume that a continuum description of the chain is valid, and derive the simplest form of the associated Hamiltonian. We will also assume that ground state dominance holds.

The partition function of the hydrophilic hydrophobic chain reads

$$
Z=\int \prod_{i} d \vec{r}_{i} e^{-\frac{d}{2 a^{2}} \sum_{i}\left(\vec{r}_{i+1}-\vec{r}_{i}\right)^{2}-\beta \mathcal{H}}
$$

where $a$ is a typical monomer length and $i=1,2, \ldots N$. The lattice Hamiltonian $(\mathcal{H})$ has been derived in equation (3), and its off-lattice version reads

$$
\begin{aligned}
\beta \mathcal{H}= & \frac{1}{2} \sum_{i \neq j}\left[v_{0}+\beta\left(\lambda_{i}+\lambda_{j}\right)\right] \delta\left(\vec{r}_{i}-\vec{r}_{j}\right) \\
& +\frac{1}{6} \sum_{i \neq j \neq k} w_{0} \delta\left(\vec{r}_{i}-\vec{r}_{j}\right) \delta\left(\vec{r}_{j}-\vec{r}_{k}\right) \\
& +\frac{1}{24} \sum_{i \neq j \neq k \neq l} y_{0} \delta\left(\vec{r}_{i}-\vec{r}_{j}\right) \delta\left(\vec{r}_{j}-\vec{r}_{k}\right) \delta\left(\vec{r}_{k}-\vec{r}_{l}\right)
\end{aligned}
$$

Note that we have also included three $\left(w_{0}\right)$ and four $\left(y_{0}\right)$ body terms for reasons that will soon become clear. We also assume $\left(\vec{r}_{1}=\vec{r}_{N}=\overrightarrow{0}\right)$.

Defining the local density $\rho(\vec{r})$ as

$$
\rho(\vec{r})=\sum_{i} \delta\left(\vec{r}-\vec{r}_{i}\right)
$$

we have

$$
Z=\int \mathcal{D} \phi(\vec{r}) \mathcal{D} \rho(\vec{r}) \zeta(\rho, \phi) \exp \left(i \int d^{d} r \phi(\vec{r}) \rho(\vec{r})-\int d^{d} r\left[\frac{v_{0}}{2} \rho^{2}(\vec{r})+\frac{w_{0}}{6} \rho^{3}(\vec{r})+\frac{y_{0}}{24} \rho^{4}(\vec{r})\right]\right)
$$


with

$$
\zeta(\rho, \phi)=\int \prod_{i} d \vec{r}_{i} e^{-\frac{d}{2 a^{2}} \sum_{i}\left(\vec{r}_{i+1}-\vec{r}_{i}\right)^{2}-i \sum_{i} \phi\left(\vec{r}_{i}\right)-\beta \sum_{i} \lambda_{i} \rho\left(\vec{r}_{i}\right)}
$$

For the periodic hydrophilic hydrophobic chain, we introduce transfer matrices $T_{ \pm}$for $\lambda_{i}=$ \pm 1 and get

$$
\zeta(\rho, \phi)=<\overrightarrow{0}\left|\left[T_{+}^{p} T_{-}^{p}\right]^{\frac{N}{2 p}}\right| \overrightarrow{0}>
$$

with

$$
<\vec{r}\left|T_{+}\right| \vec{r}^{\prime}>=e^{-\frac{d}{2 a^{2}}\left(\vec{r}-\vec{r}^{\prime}\right)^{2}-i \phi(\vec{r})-\beta \rho(\vec{r})}
$$

and

$$
<\vec{r}\left|T_{-}\right| \vec{r}^{\prime}>=e^{-\frac{d}{2 a^{2}}\left(\vec{r}-\vec{r}^{\prime}\right)^{2}-i \phi(\vec{r})+\beta \rho(\vec{r})}
$$

In equation (13), the differences between $p \sim O(N)$ and $p \sim O(1)$ are clearly displayed. From now on, we will set $p=1$, without questioning any further the existence of the continuum limit in this case. Using the identity

$$
<\vec{r}\left|T_{+} T_{-}\right| \vec{r}^{\prime}>=\int d^{d} r_{0}<\vec{r}\left|T_{+}\right| \vec{r}_{0}><\vec{r}_{0}\left|T_{+}\right| \vec{r}^{\prime}>
$$

together with equations (14) (15) leads, to lowest non trivial order in $\phi(\vec{r})$ and $\rho(\vec{r})$, to

$$
<\vec{r}\left|T_{+} T_{-}\right| \vec{r}^{\prime}>=e^{-\frac{d}{4 a^{2}}\left(\vec{r}-\vec{r}^{\prime}\right)^{2}-2 i \phi(\vec{r})+\frac{a^{2}}{4 d} \beta^{2}(\vec{\nabla} \rho)^{2}(\vec{r})}
$$

which implies

$$
\zeta(\rho, \phi)=\operatorname{Tr} \exp -\left(\frac{N}{2} \mathcal{H}_{0}\right)
$$

with

$$
\mathcal{H}_{0}=-\frac{a^{2}}{4 d} \vec{\nabla}^{2}+2 i \phi(\vec{r})-\frac{a^{2}}{4 d} \beta^{2}(\vec{\nabla} \rho)^{2}(\vec{r})
$$

It is quite clear that our derivation is not rigorous. We nevertheless feel that the $\rho(\vec{r})$ dependent term of the Hamiltonian $\mathcal{H}_{0}$ is physically sound since it favors inhomogeneous high 
density regions (droplets). A better approximation would presumably involve higher derivatives of $\rho(\vec{r})$, which clearly define typical droplet sizes. Assuming ground state dominance in (19), and performing a saddle point approximation on $\phi(\vec{r})$ in (11) yields

$$
\rho(\vec{r})=N \Psi^{2}(\vec{r})
$$

where $\Psi(\vec{r})$ is a normalized wave function. We then obtain the "free energy" per monomer as

$$
\beta \frac{F}{N}=\min _{\{\Psi(\vec{r})\}} \int d^{d} r G(\Psi(\vec{r}))
$$

where

$$
\begin{aligned}
G(\Psi(\vec{r}))= & \int d^{d} r\left(\frac{v_{0} N}{2} \Psi^{4}+\frac{w_{0} N^{2}}{6} \Psi^{6}+\frac{y_{0} N^{3}}{24} \Psi^{8}\right) \\
& +\frac{1}{2} \int d^{d} r \Psi(\vec{r})\left(-\frac{a^{2}}{4 d} \vec{\nabla}^{2}-\frac{a^{2}}{4 d} \beta^{2}\left(\vec{\nabla}\left(N \Psi^{2}\right)\right)^{2}(\vec{r})\right) \Psi(\vec{r})
\end{aligned}
$$

At this point, it is useful to remark that one needs in this approximation to introduce four body interactions, as in the disordered case, and for the same reasons [4]. The fact that attractive multi-body interactions in homopolymers may induce a SAW-BP phase transition has been previously noted for a specific model in reference 30].

At low temperature, the last term of equation (22) plays a dominant role. This term, as mentionned above, tends to create an interface between hydrophilic and hydrophobic regions. If one uses a variational wave function $\Psi_{0}(\vec{r})$ given (for $d=2$ ) by

$$
\Psi_{0}(\vec{r})=a_{0} \cos \left(\frac{2 \pi x}{l}\right) \cos \left(\frac{2 \pi y}{l}\right)
$$

the normalization condition implies

$$
a_{0}^{2} R^{2} \sim O(1)
$$

where $R$ is the linear dimension of the system. Plugging this estimate in equation (22) shows that a low free energy is obtained for $l \sim a$ and a finite average density $\rho_{a v}=\frac{N}{R^{2}} \sim O(1)$. In other words, the low temperature phase obtained from our variational approach is a dense 
phase (since $\nu=\frac{1}{d}$, with $d=2$ ), made of microscopically phase separated regions. In section ( $(\mathbb{E A})$, we obtained from our simulations the value $\nu \simeq 0.64$ below the the (SAW)(BP) phase transition. This result is not compatible with the value $\nu=0.5$ that we get through the variational method. Some possible explanations for this "discrepancy" are as follows

(i) The simulations were done for $p=8$ on a lattice, and the variational method was applied to the case $p=1$, within a continuum limit approach.

(ii) A major difference between the present multi droplet collapse and the $\theta$ single core collapse is that one has to take into account the degeneracy of the saddle point equation for $\phi(\vec{r})$ in evaluating the "true" free energy. In other words, there is a droplet entropy that must be considered. Sticking with the variational function of equation (23), it is easily seen that this entropy favors large values of $\frac{l}{a}$. A precise calculation is difficult, and we will not comment upon this point anymore.

(iii) We believe however that the main reason for the difference between the exponents $\nu$ stems from the use of a ground state approximation in estimating the right handside of equation (18). As far as we know, this approximation, which relies on the existence of a bound state in the Hamiltonian $\mathcal{H}_{0}$, works well for dense (finite density) phases. It does not a priori describe a branched polymer, which has a vanishing density. Physically, a dense phase is not favorable because of the repulsion between the hydrophilic monomers.

Altogether, our results seems to indicate that the finite $p$ chain may undergo zero $(p=4)$, one $(p=8)$ or two $(p=10,12)$ phase transitions. Ground state dominance is never a valid approximation, since one deals with either linear $(\nu=0.75)$ or branched $(\nu \simeq 0.64)$ structures. Reentrant behaviour, similar to the one described for $p=10,12$, has been found recently in related models [31,32. Another issue of interest concerns a possible low temperature dynamical (glass) transition, similar to the one described in reference [8]. 


\section{CONCLUSION}

We have studied a periodic hydrophilic hydrophobic chain. An important ingredient of the physics of this problem is the value of the period $2 p$. The low temperature phase consists of a single $\left(p>p_{c}(d, N)\right)$ or multiple $\left(p<p_{c}(d, N)\right)$ hydrophobic core(s), where

$p_{c}(3, N) \sim O\left(N^{\frac{1}{3}}\right)$ and $p_{c}(2, N) \sim O(N)$. Using Monte Carlo calculations, we have studied the case $p \sim O(N)$ and indeed found a macroscopic phase separation between the two types of monomers in $d=3$, and two possible regimes in $d=2$. The second case, $(p \sim O(1))$ yields for both dimensions a low temperature phase, consisting of a chain or network of microscopic hydrophobic droplets linked by hydrophilic filaments. We have studied this phase numerically in $d=2$. A connection with both the branched polymer chain [27] and the random hydrophilic hydrophobic chain [4,5,6,07,31] is physically appealing and shows up in the tentative variational treatment of this phase, as given in section $\mathbb{E C}$. As for proteins, the existence of periodic hydrophobicity patterns in secondary structures [10] suggests that our model may have some relevance in explaining the typical size of single domain proteins ( $N \sim 120-150$ residues). Further work in these directions is in progress.

It is a pleasure to thank Henri Orland for fruitful discussions and suggestions, and Bernard Derrida for interesting comments. 


\section{REFERENCES}

[1] T. Garel, H. Orland and E. Pitard, "Protein folding and heteropolymers", in Spin Glasses and Random Fields, A.P. Young (ed.), World Scientific, Singapore (1997) p. 387-443.

[2] V.R. Agashe, M.C.R. Shastry and J.B. Udgaonkar, Nature, 377, 754 (1995).

[3] S.P. Obukhov, J. Phys. A, 19, 3655 (1986).

[4] T. Garel, L. Leibler and H. Orland, J. Phys. (France) II, 4, 2139 (1994).

[5] D. Thirumalai, V. Ashwin and J.K. Bhattacharjee, Phys. Rev. Lett., 77, 5385 (1996).

[6] A. Moskalenko, Y.A. Kuznetsov and K.A. Dawson, J. Phys. (France), II, 7, 409 (1997).

[7] A. Moskalenko, Y.A. Kuznetsov and K.A. Dawson, Europhys. Lett., 40, 135 (1997).

[8] T.R. Kirkpatrick, D. Thirumalai and P.G. Wolynes, Phys. Rev. A, 40, 1045 (1989).

[9] Hao Li, C. Tang and N.S. Wingreen, Phys. Rev. Lett., 79, 765 (1997).

[10] A. Irbäck, C. Peterson and F. Rotthast, Proc. Natl. Acad. Sci. (USA), 93, 9533 (1996).

[11] E. Orlandini “ Monte Carlo Study of Polymer Systems by Multiple Markov Chain Method”,to appear in "Numerical methods for Polymeric Systems", ed. S. Whittington, IMA Volumes in Mathematics and its Applications, (Springer Verlag 1998).

[12] M. C. Tesi, E. J. Janse van Rensburg, E. Orlandini and S. G. Whittington, J. Stat. Phys., 29, 2451 (1996).

[13] N. Madras and A. D. Sokal, J. Stat. Phys. 47, 573 (1987).

[14] P. H. Verdier and W. H. Stockmayer, J. Chem. Phys., 36, 227 (1961).

[15] A. Milchev, W. Paul and K. Binder, J. Chem. Phys., 99, 4786 (1993). 
[16] M. Wittkop, S. Kreitmer and D. Göritz, J. Chem. Phys., 104, 3373 (1996).

[17] A. Baumgärtner, J. Phys. (Paris), 43, 1407 (1982).

[18] H. Meirovitch and H.A. Lim, J. Chem. Phys., 91, 2544 (1989).

[19] P.Grassberger and R. Hegger, J. Phys. I (France), 5, 597 (1995).

[20] P.Grassberger and R. Hegger, J. Chem. Phys., 102, 6881 (1995).

[21] B. Duplantier and H. Saleur, Phys. Rev. Lett., 59, 539 (1987).

[22] J.C. Le Guillou and J. Zinn-Justin, J. Phys. (France), 50, 1365 (1989).

[23] R. Guida and J. Zinn-Justin, Saclay preprint SPhT /97 - 040.

[24] M. Dadmun and M. Muthukumar, J. Chem. Phys., 97, 578 (1992).

[25] P.M. Lam, Phys. Rev. B, 36, 6988 (1987).

[26] B. Nienhuis, Phys. Rev. Lett,, 49, 1063 (1982).

[27] T.C. Lubensky and Joel Isaacson, Phys. Rev. A, 20, 2130 (1979).

[28] B. Derrida and L. de Sèze, J. Phys. (France), 43, 475 (1982).

[29] G. Parisi and N. Sourlas, Phys. Rev. Lett., 46, 871 (1981).

[30] E. Orlandini, F. Seno, A.L. Stella and M.C. Tesi, Phys. Rev. Lett., 68, 488 (1992).

[31] A. Trovato, J. van Mourik and A. Maritan, Swollen-Collapsed Transitiom in Random Heteropolymers, preprint cond-mat/9804098.

[32] D.E. Leckband, O.V. Borisov and A. Halperin, Macromolecules, 31, 2368 (1998). 


\section{Figure captions}

Figure 1: Scaled radius of gyration $\left(\frac{<R^{2}>}{N^{2} \nu_{\theta}}\right)$ vs temperature for the purely hydrophobic chain in $d=2$, for $N=80(\triangle), 160(\square), 240(\diamond), 480(\times), 640(+)$. A crossing occurs for $T_{\theta} \simeq 1.5$.

Figure 2: Specific heat vs temperature, for the purely hydrophobic chain in $d=2$, for the same values of $N$. The extrapolated critical temperature is $T_{\theta}^{\prime} \simeq 1.5$.

Figure 3: Typical phase separated configuration $\left(d=3, p=\frac{N}{8}, N=640\right)$. Circles denote hydrophobic monomers.

Figure 4: Log-Log plot of $<R^{2}>$ vs $N$ at various temperatures for $\left(d=3, p=\frac{N}{8}\right)$, and $N=80,160,240,360,480,640,800$. The temperature is $T=\infty(\square), 3.33(\triangle), 2.0(*)$,

,1.0 $(\diamond), 0.5(+)$. The upper and lower straight lines have slopes compatible with self avoiding behaviour $2 \nu_{S A W} \simeq 1.176$.

Figure 5: Specific heat vs temperature for $\left(d=3, p=\frac{N}{8}\right)$, and $N=80(\mathrm{O}), 160(\square)$, $240(\diamond), 360(\times), 480(+), 640(\triangle)$. Note the increase of the peak as well as its shape, when $N$ increases.

Figure 6: (a) Plot of the specific heat peak $C^{*}$ vs $N$ for $\left(d=3, p=\frac{N}{8}\right)$, and $N=$ 80, 160, 240, 360, 480, 640 (b) Plot of the critical temperature vs $\frac{1}{N}$ for the same parameters. Error bars correspond to one standard deviation.

Figure 7: Scaled phase separation parameter (see equation (7)) vs temperature, for $\left(d=3, p=\frac{N}{8}\right)$, and $N=80,160,240,360,480,640$.

Figure 8: Low temperature phase separated configuration $\left(d=2, p=\frac{N}{8}, N=240\right)$. Black triangles denote hydrophilic monomers. Note the isotropic shape of the hydrophobic core $\left(p>>p_{c}\right)$.

Figure 9: Log-Log plot of $<R^{2}>$ vs $N$ at various temperatures for $\left(d=2, p=\frac{N}{8}\right)$, and $N=80,160,240,360,480,640,800$. The temperature is $T=\infty(\square), 1.4(\triangle), 1.0(*), 0.5(\diamond)$. The upper and lower straight lines have slopes compatible with self avoiding behaviour $2 \nu_{S A W}=1.5$. 
Figure 10: Low temperature multiple cores' configuration displaying the screening effect of the hydrophilic loops $\left(d=2, p=\frac{N}{24}, N=240\right)$.

Figure 11: Low temperature phase separated configuration $\left(d=2, p=\frac{N}{12} \simeq p_{c}+\right.$, $N=240)$. Note the elongated shape of the hydrophobic core.

Figure 12: Typical multiple cores' configuration $(d=2, p=8, N=1200)$. Black triangles denote hydrophilic monomers.

Figure 13: $\log -\log$ plot of $<R^{2}>$ vs $N$ at various temperatures for $(d=2, p=8)$, and $N=80,160,240,360,480,640,800,1200$. The temperature is $T=\infty(\square), 1.0(\triangle), 0.7(*)$, ,0.5 $(\diamond), 0.33(+)$. The upper straight line has a slope $2 \nu_{S A W}=1.5$, whereas the middle line has slope $2 \nu_{B P}$, where $\nu_{B P} \simeq 0.64$ is the branched polymer (BP) value. The bottom line corresponds to the collapsed value $2 \nu=1$.

Figure 14: Scaled radius of gyration $\left(\frac{<R^{2}>}{N^{2 \nu^{*}}}\right)$ vs temperature for $(d=2, p=8)$ and $N=80(\square), 160(\diamond), 240(\times), 480(+), 640(\triangle)$. A crossing occurs for $\nu^{*} \simeq 0.70$, yielding $T_{c} \simeq 0.8$.

Figure 15: With the same data, no crossing occurs for (a) $\nu^{*}=\nu_{S A W}=0.75$ (b) $\nu^{*}=\nu_{\theta}=\frac{4}{7}(\mathrm{c}) \nu^{*}=\nu_{B P} \simeq 0.64$.

Figure 16: Typical multiple cores' configuration $(d=3, p=4, N=720)$. Circles denote hydrophobic monomers. 


\section{Table caption}

Table I: Comparison between the critical temperature of the hydrophilic hydrophobic chain $T_{c}=T_{c}(p, N)$, and the $\theta$ transition temperatures [12] of a fully hydrophobic chain of (i)

$p$ monomers (ii) $\frac{N}{2}$ monomers. The first three lines correspond to $p=\frac{N}{8}(N=80,640,800)$. The last line corresponds to $p=\frac{N}{4}(N=800)$. The value $T_{\theta}(p=10)$ has been obtained using exact enumeration techniques. 


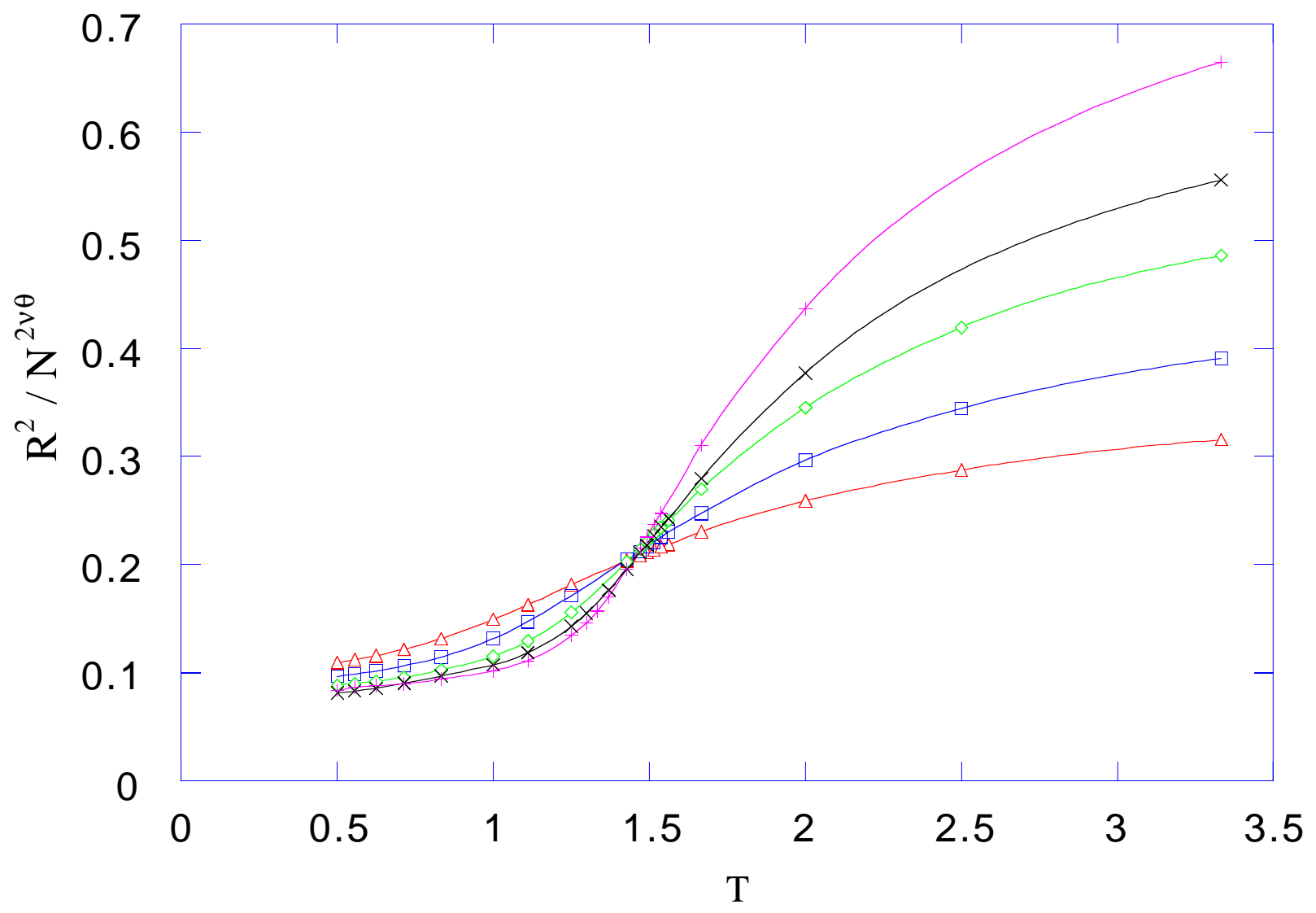




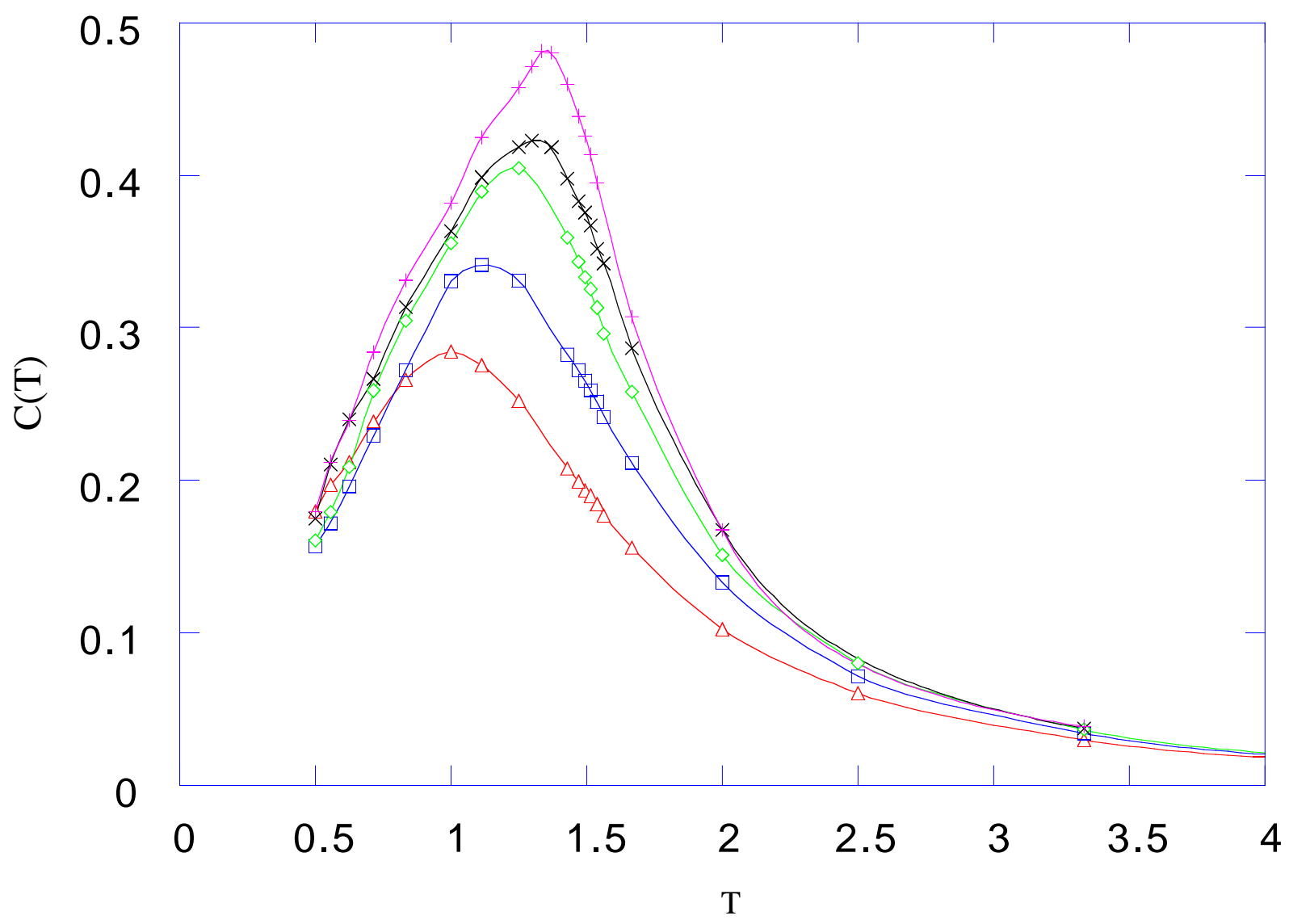




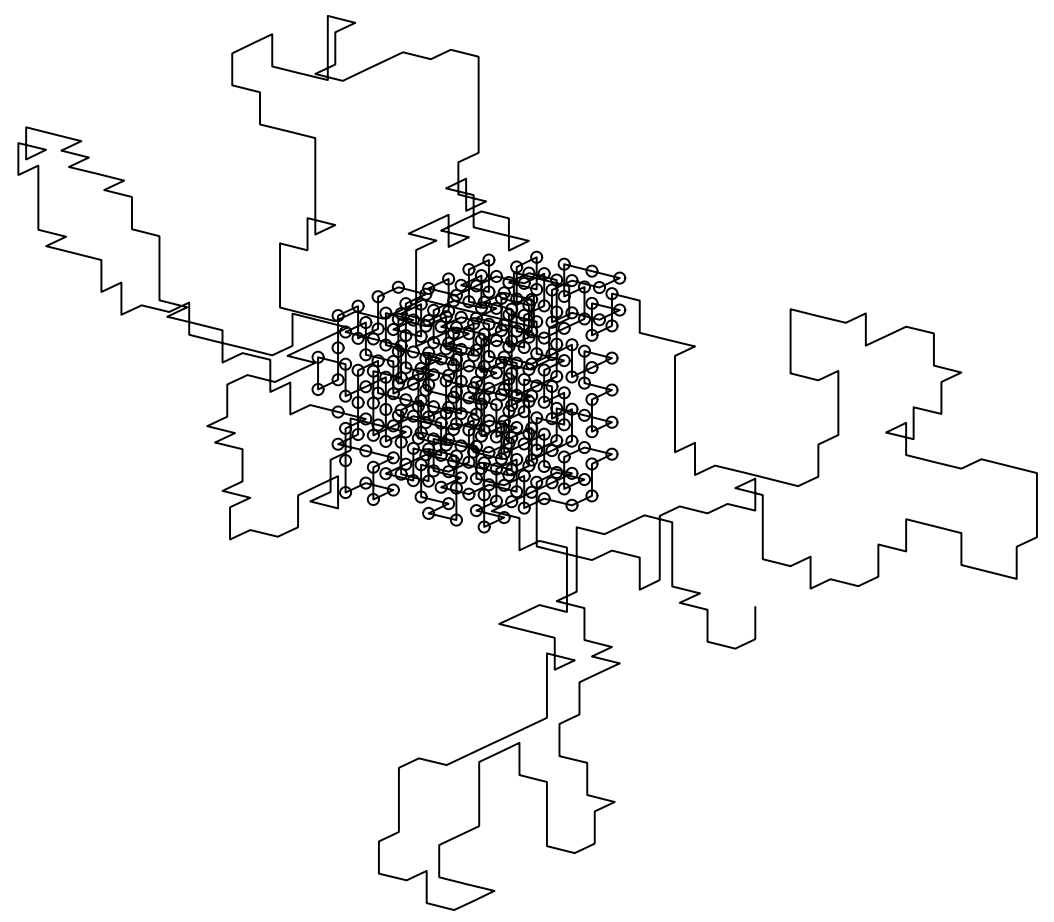




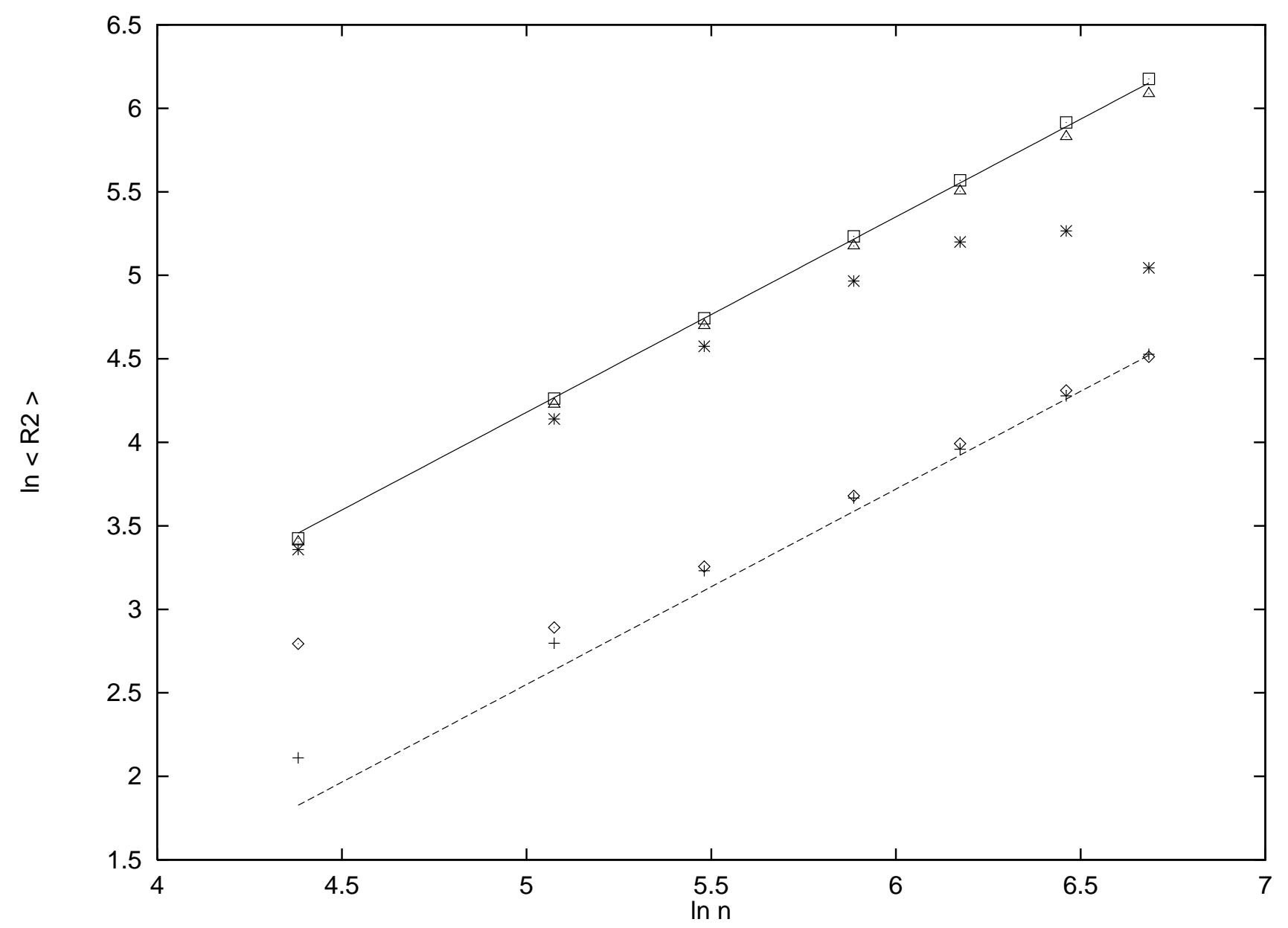




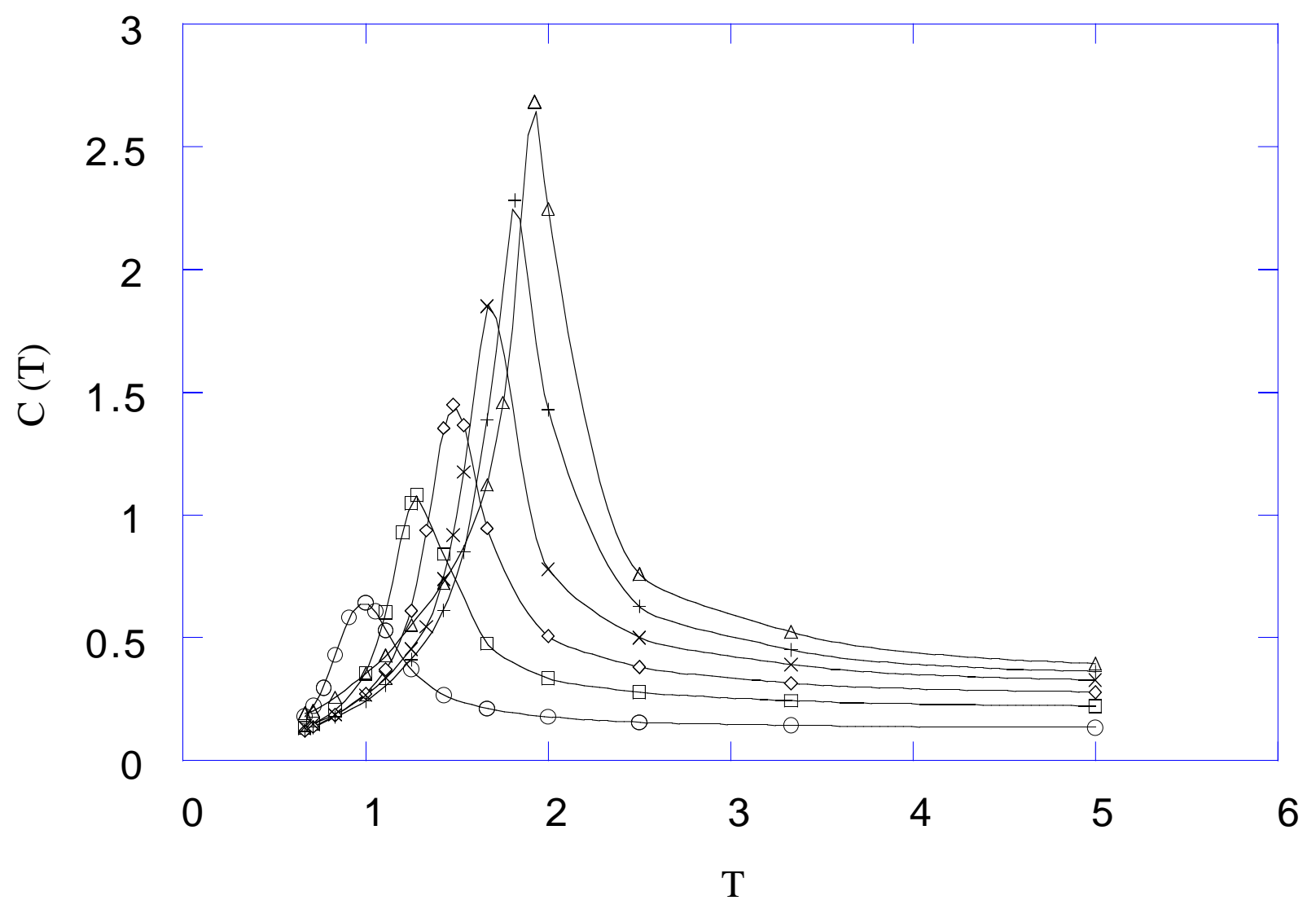


(a)

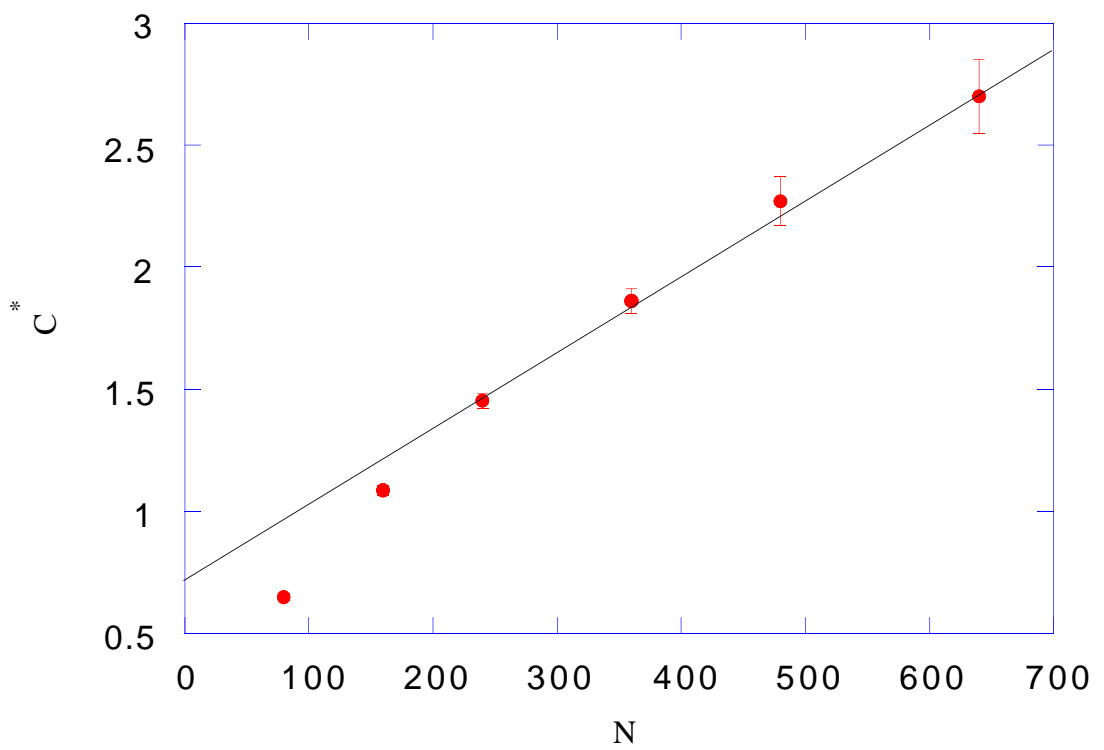

(b)

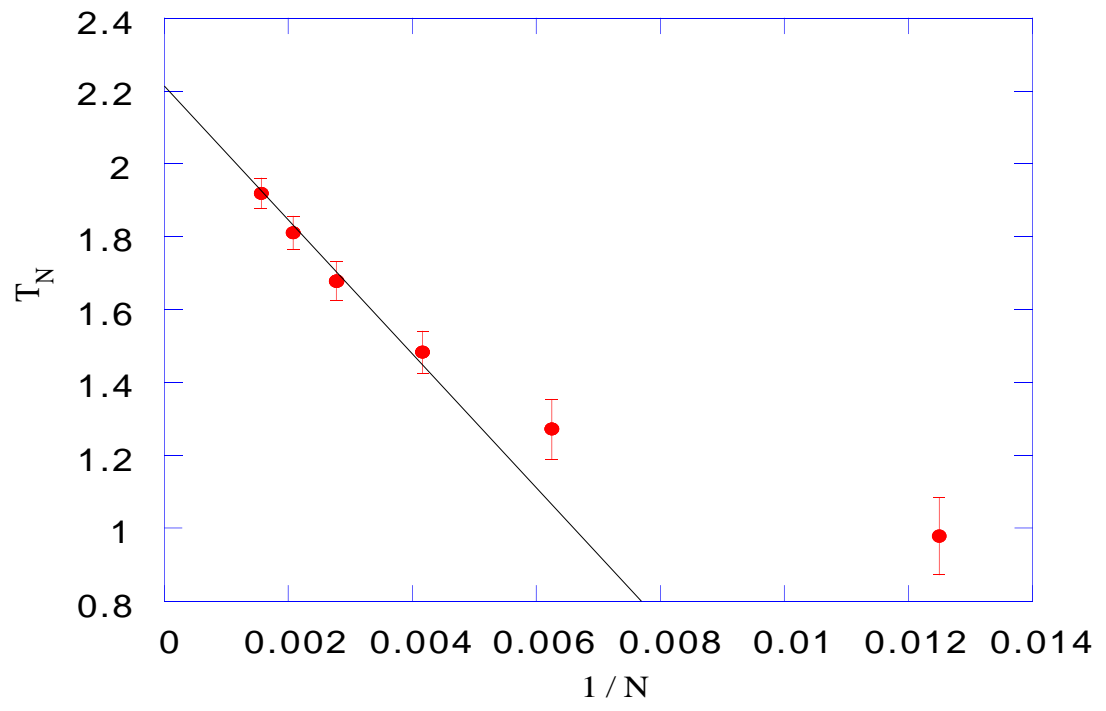




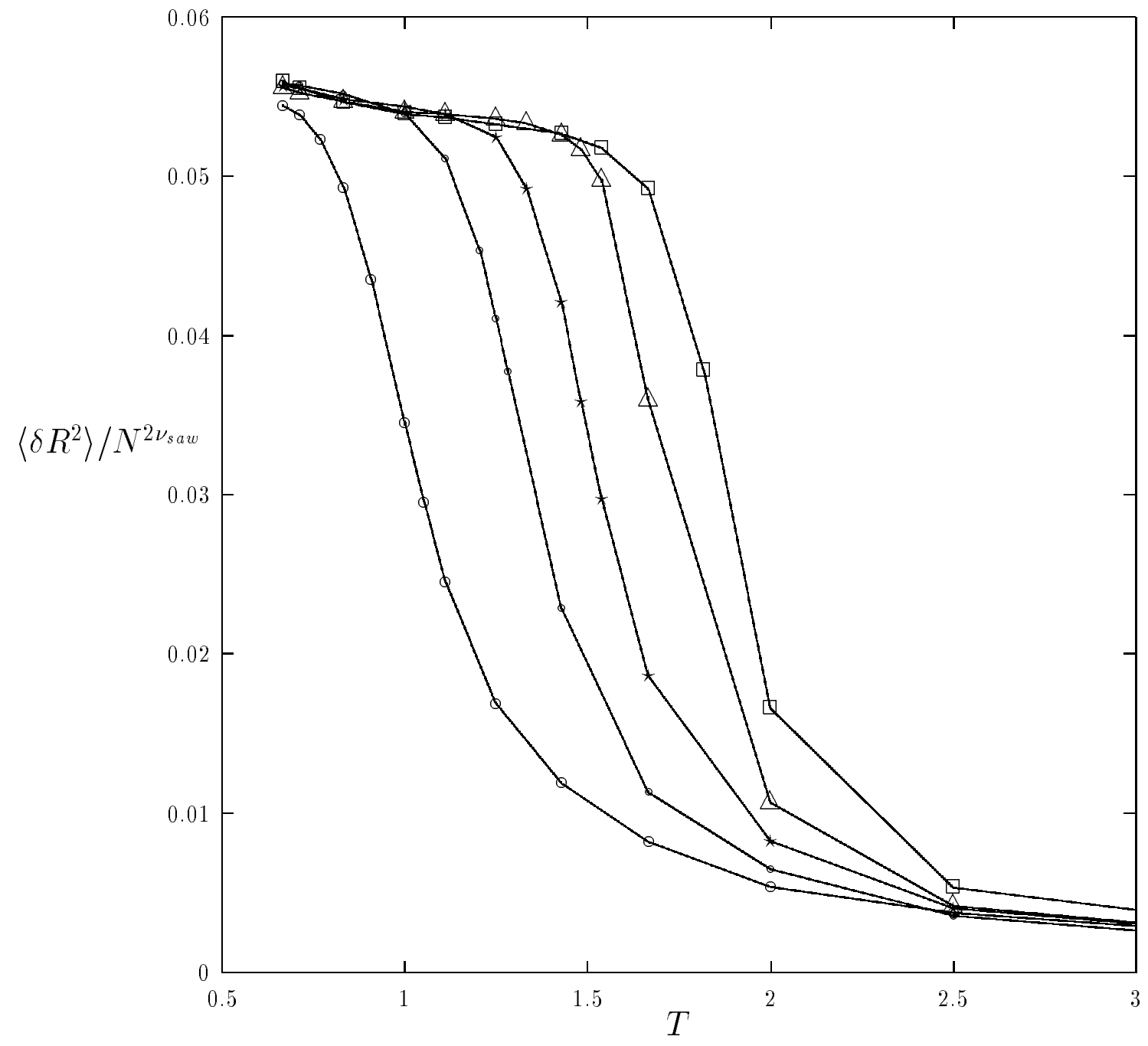




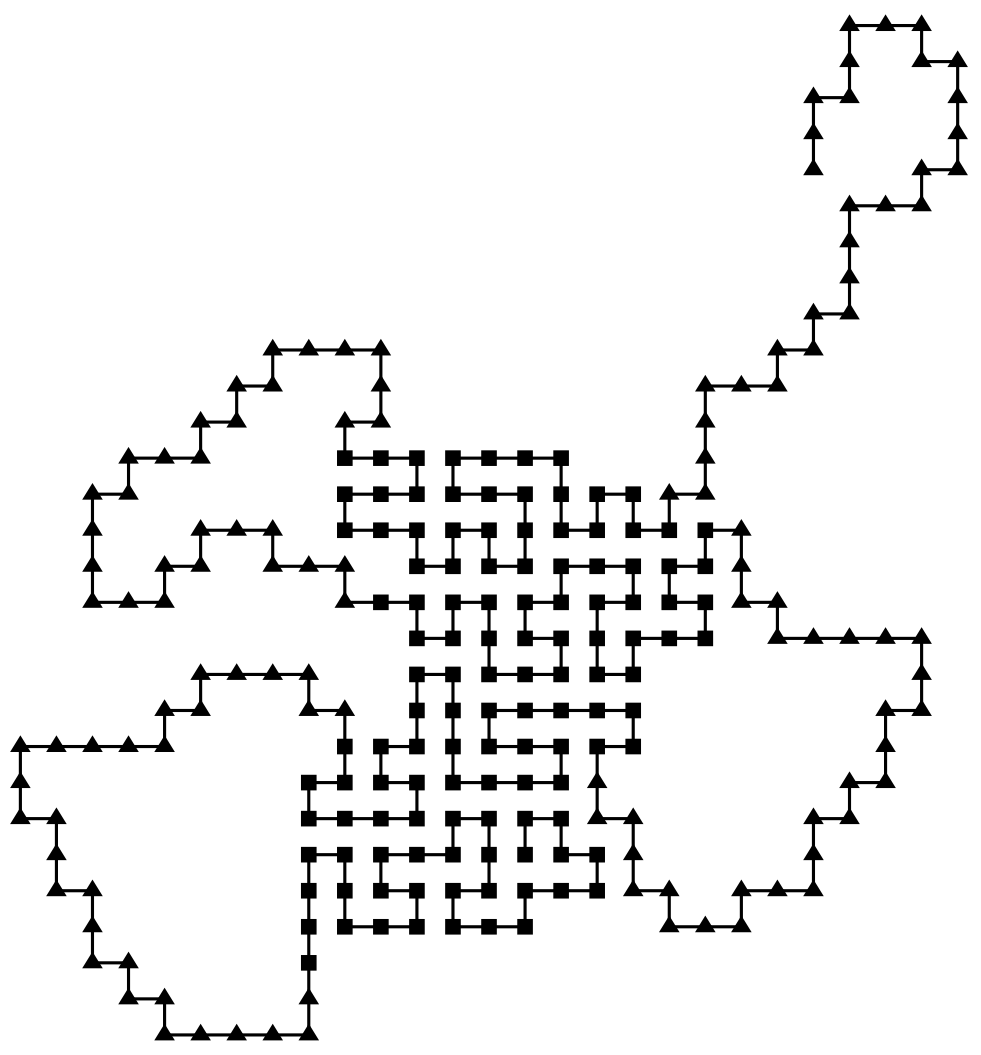




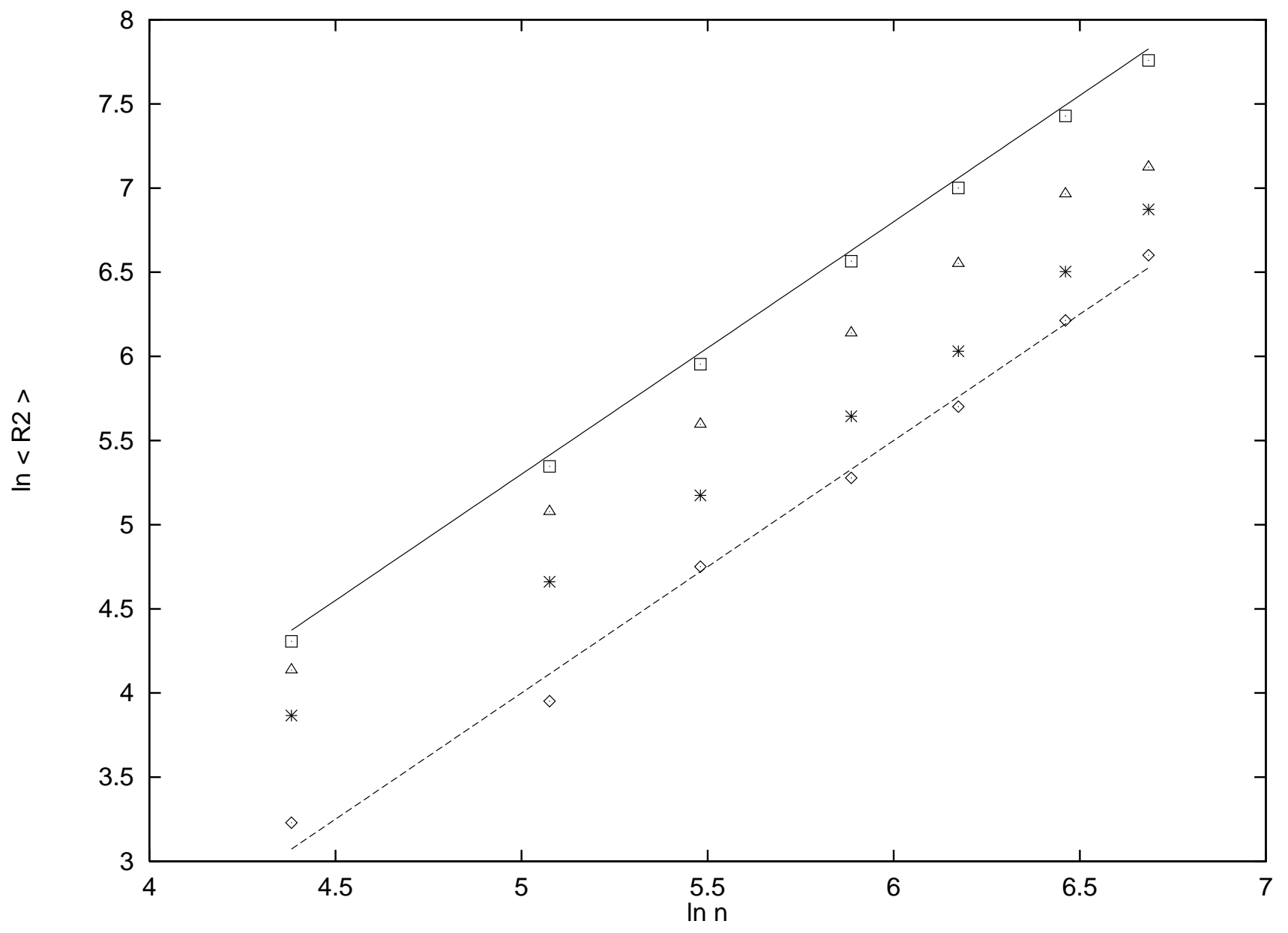




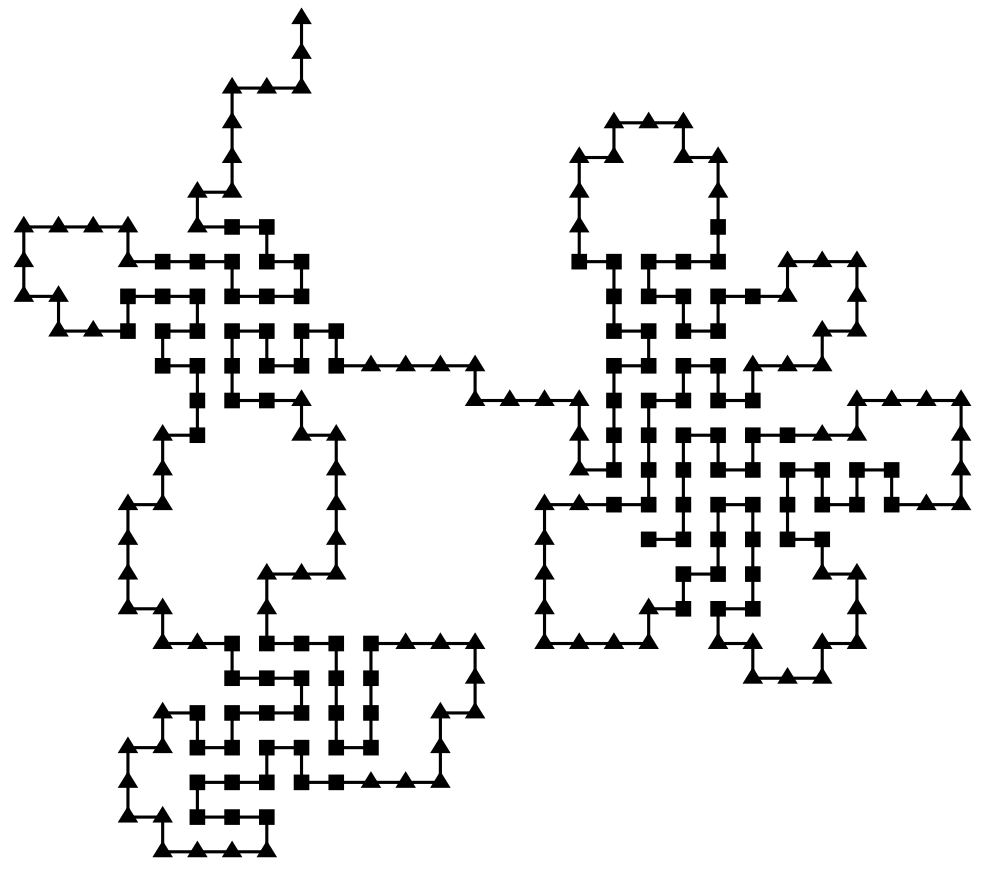




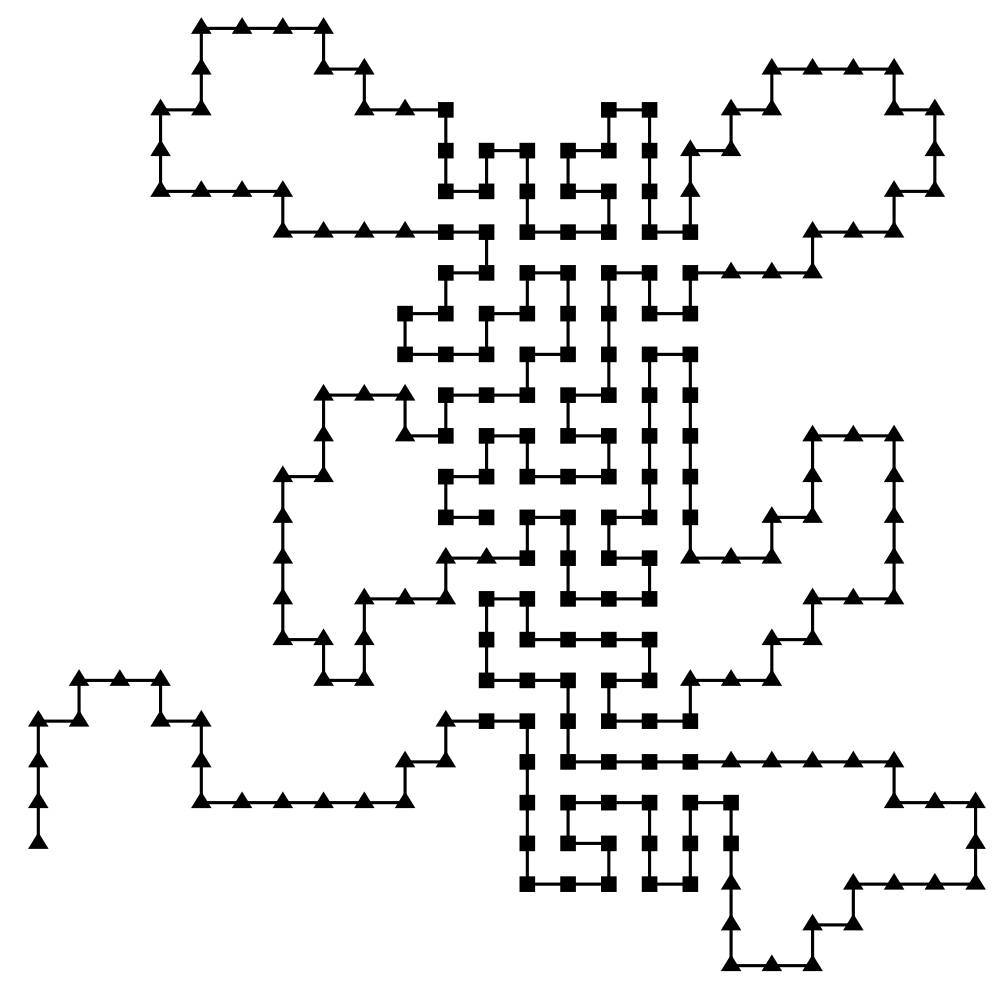




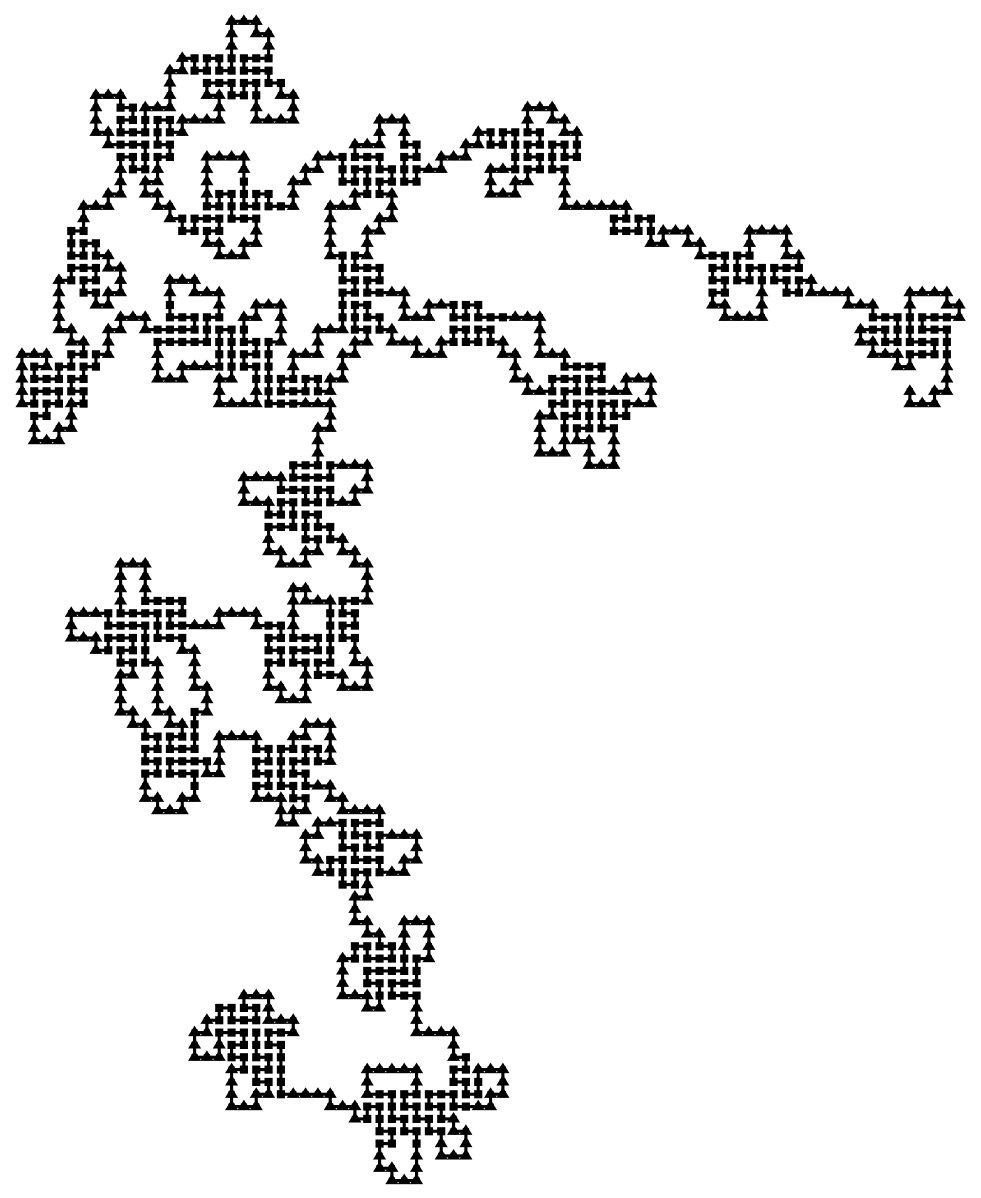




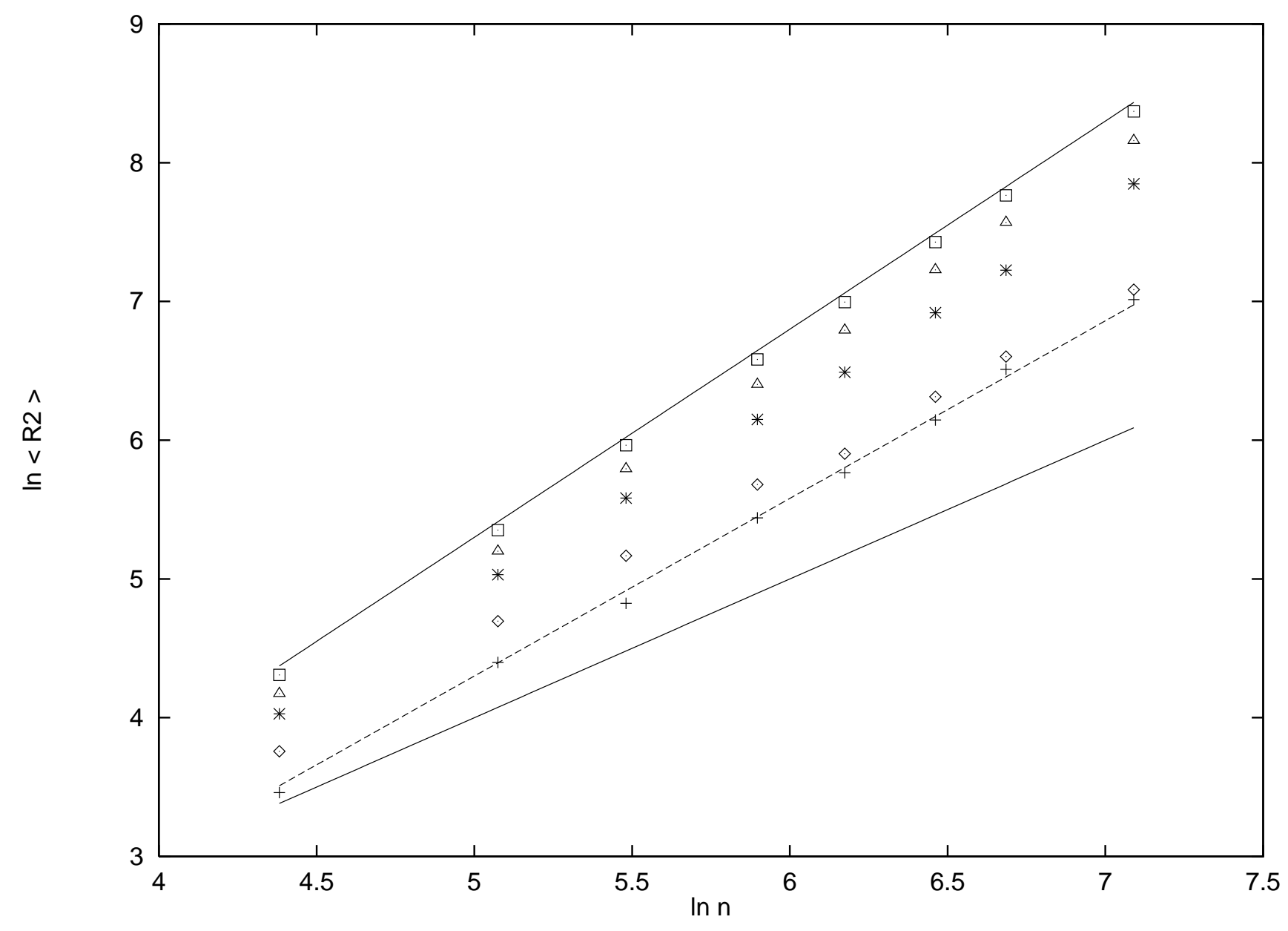




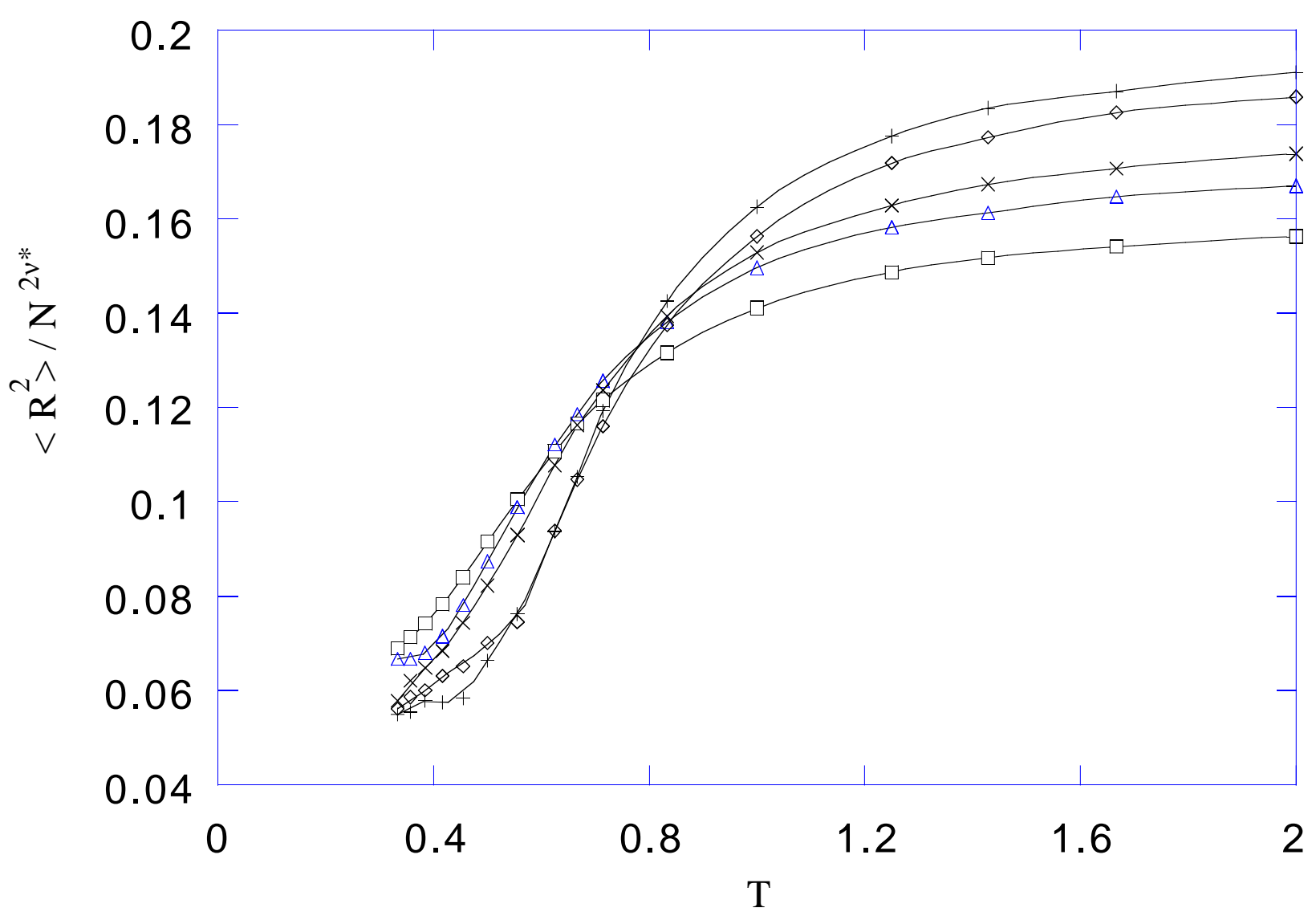


(a)
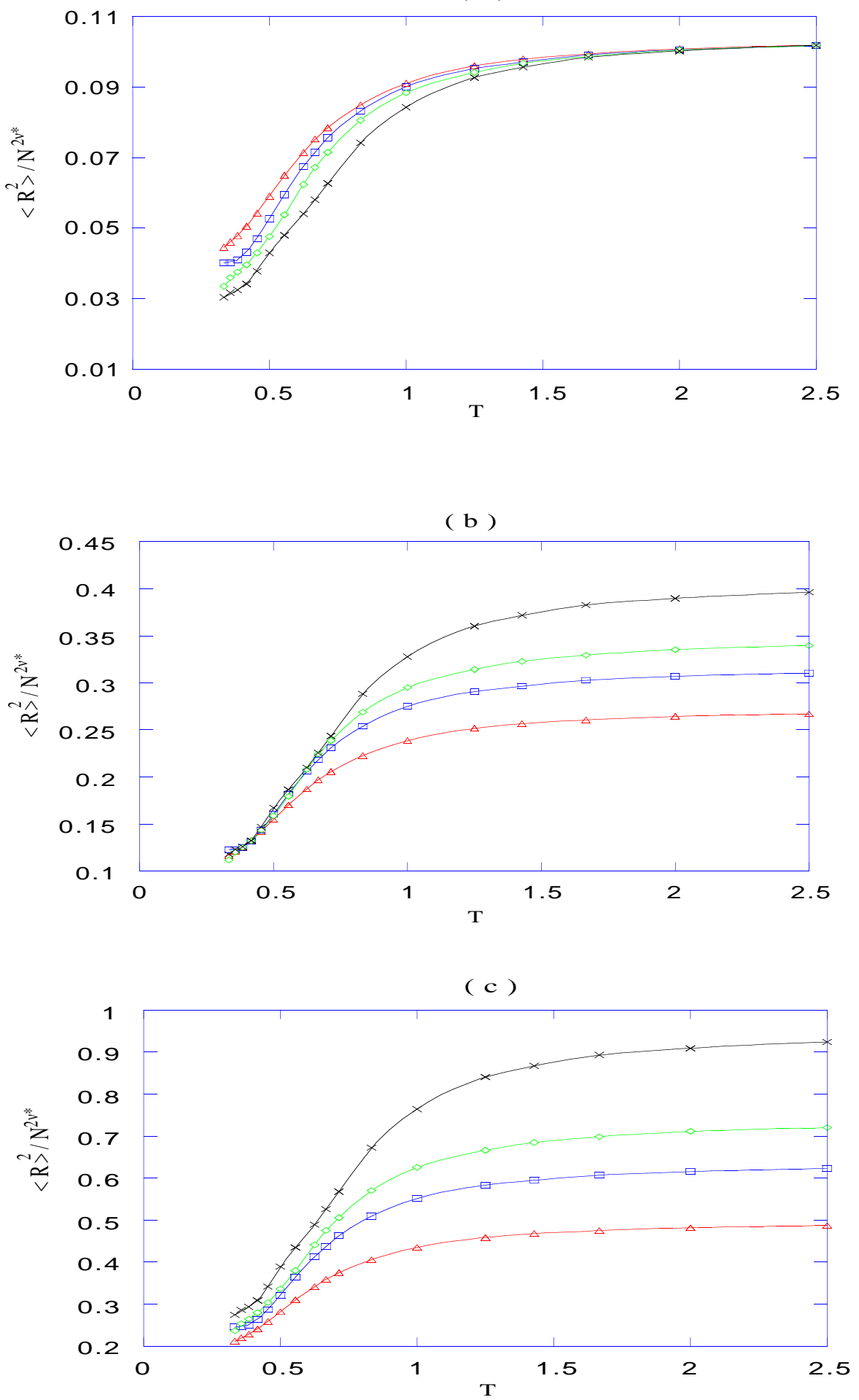


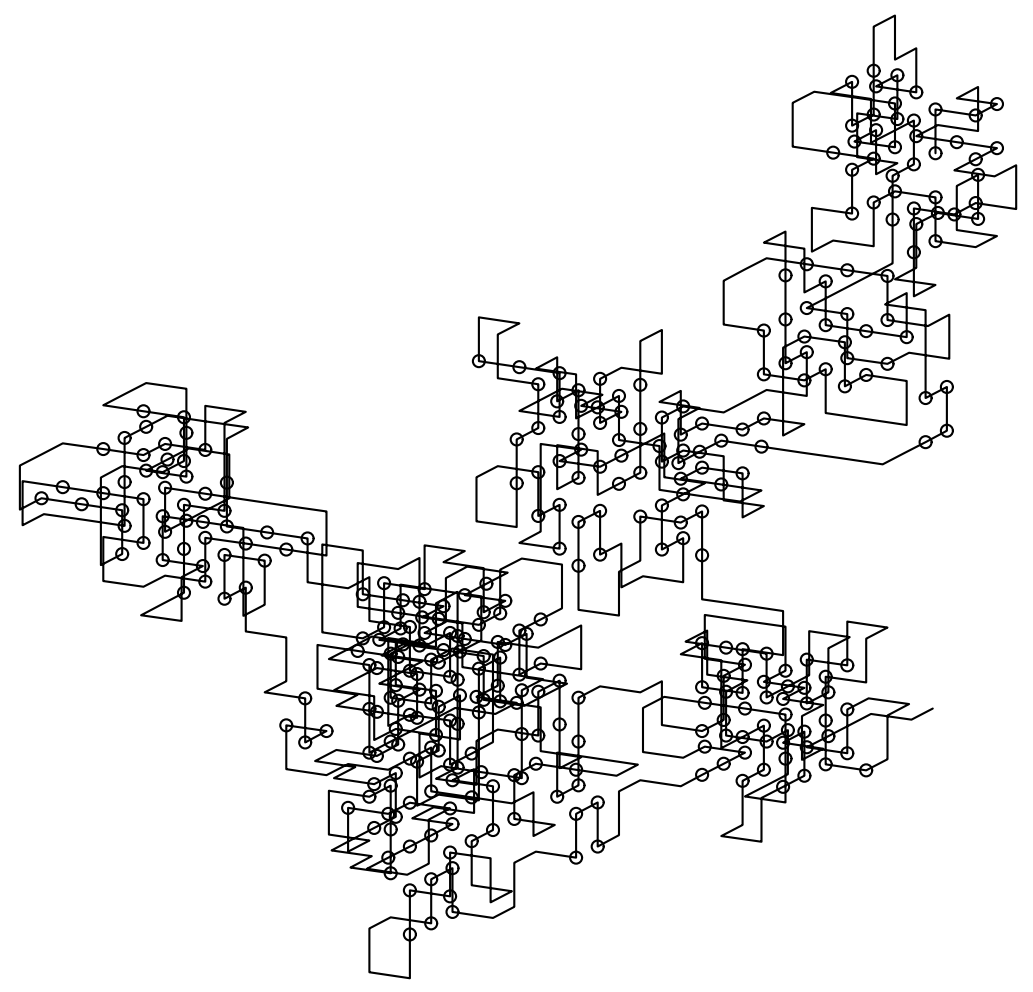




\begin{tabular}{|r|r|r|}
\hline$T_{c}$ & $T_{\theta}(p)$ & $T_{\theta}(N / 2)$ \\
\hline $0.98 \pm 0.07$ & 1.087 & $1.78 \pm 0.03$ \\
$1.92 \pm 0.11$ & $2.08 \pm 0.08$ & $2.51 \pm 0.12$ \\
$2.08 \pm 0.15$ & $2.13 \pm 0.14$ & $2.61 \pm 0.14$ \\
$2.27 \pm 0.16$ & $2.38 \pm 0.14$ & $2.61 \pm 0.14$ \\
\hline
\end{tabular}

